\title{
Factores pronósticos en pacientes pluripatológicos de edad avanzada en un hospital de asistencia a crónicos de media y larga estancia (HACMLE)
}

\author{
R. NAVARRO SANZ, P. JUEZ MARTEL ${ }^{1}$, V. SANCHIS-BAYARRI BERNAL, \\ E. CASTELLANO VELA
}

Área Médica Integral - Medicina Interna. Hospital Pare Jofré. Agencia Valenciana de Salud. ${ }^{1}$ UNED. Madrid

\begin{abstract}
PROGNOSTIC FACTORS IN ELDERLY PATIENTS WITH CO-OCCURRING DISEASES IN A MEDIUM-LONG-TERM AND CHRONIC CARE HOSPITAL (MLT\&CCH)
\end{abstract}

\section{RESUMEN}

Objetivo: Conocer los factores pronósticos de mortalidad hospitalaria en los pacientes ancianos y pluripatológicos ingresados en nuestro Hospital de Asistencia a Crónicos de Media y Larga Estancia.

Método: Se realiza una valoración multidimensional de forma longitudinal y prospectiva a 117 pacientes mayores de 75 años, tanto al ingreso como al alta o fallecimiento y se le aplica un tratamiento estadístico (Modelo de Regresión Logística).

Resultado: Se aprecia un predominio significativo de mujeres sobre hombres, una elevada prevalencia de deterioro neurológico, y especialmente un alto porcentaje de pacientes que ingresaba con dos o más factores de riesgo. Comprobamos como el deterioro cognitivo, la situación funcional y los niveles bajos de albúmina, se asocian significativamente con una mayor mortalidad.

Conclusiones: La evaluación de estas variables es fundamental para emitir un pronóstico y en ocasiones, para mejorarlo. Nuestros resultados, en líneas generales, son similares a la literatura revisada.

PALABRAS CLAVE: Ancianos hospitalizados. Pluripatológicos. Factores pronósticos. Mortalidad.

\section{ABSTRACT}

Objective: To know the prognostic factors for hospitalary mortality in elderly patients with co-occuring diseases in our medium-long-term and chronic care Hospital (MLT\&CCH).

Method: We performed longitudinally and prospectively a multiple assessment to 117 patients aged 75 or older. This assessment was made at inclusion and discharge or die, and data were studied by statistical analysis (Logistic Regression Model).

Results: The prevalence of women over men was significative. We also detected a high prevalence of neurologic impairment, but mainly the high percentage of admitted patients with two or more risk factors. We appreciate that cognitive dilapidation, social disability and low levels of albumine were significantly associated with higher mortality.

Conclusions: The evaluation of these variables is fundamental to emit a prognosis and some times to improve it. Our results mainly concur with previous reports in literature.

KEY WORDS: Hospitalized elderly. Comorbidity. Prognostic factors. Mortality.

Navarro Sanz, R, Juez, Martel P, Sanchis-Bayarri Bernal V, Castellano Vela E. Factores pronósticos en pacientes pluripatológicos de edad avanzada en un hospital de asistencia a crónicos de media y larga estancia (HACMLE). An Med Interna (Madrid) 2006; 23: 529-532.

\section{INTRODUCCIÓN}

Los pacientes pluripatológicos de edad avanzada (PEAP) presentan una serie de características, como son: pluripatología, inestabilidad clínica, dependencia funcional, alteraciones cognitivas y situación socio-familiar y del entorno deprimida o crítica, que muchas veces se presentan de forma simultánea y hacen que su manejo (asistencia y gestión de recursos) tenga una especial dificultad, sobre todo para definir el pronóstico funcional e incluso vital (1). Conocemos instrumentos que intentan predecir el pronóstico a corto plazo de pacientes críticos hospitalizados, como son el APACHE III utilizado en las Unidades de Cuidados Intensivos (UCI), el SUPPORT, para establecer el pronóstico a seis meses de pacientes hospitalizados tanto dentro como fuera de las UCI, y más recientemente la versión corta del EORTC QLQ-30 para uso en cuidados paliativos (2). Conocemos estudios en pacientes con enfermedades crónicas de un órgano (insuficiencia cardíaca, accidente vascular cerebral, etc.), que evidencian la existencia de marcadores específicos de mal pronóstico (3). También es conocida la existencia de estudios que determinan marcadores generales de mal pronóstico en las personas mayores (hipoalbuminemia, hipocolesterolemia, dependencia para actividades de la vida diaria, etc.) (4).

La predicción constituye una de las actividades asistenciales y de investigación clínica con mayor auge en nuestros días (5). La mayoría de las variables de predicción en los PEAP se pueden obtener tras una adecuada valoración multidimensional (VMD) (médica: diagnósticos; funcional: dependencia para las actividades básicas e instrumentales de la vida diaria,

Trabajo aceptado: 26 de junio de 2006 
nivel cognitivo y estado emocional y demográfico: situación sociofamiliar). Los instrumentos de predicción en PEAP están dirigidos a evaluar situaciones como: envejecimiento satisfactorio, mejoría funcional, calidad de vida, aparición de síndromes geriátricos, evolución hacía la incapacidad, morbilidad, mortalidad hospitalaria, etc.

En la literatura médica existen pocos estudios sobre los factores pronósticos de mortalidad hospitalaria en PEAP, por lo que nos plateamos conocer los factores pronósticos relacionados con una mayor probabilidad de fallecimiento durante la estancia hospitalaria en pacientes ancianos, de 75 o más años de edad, con comorbilidad y discapacidad tras la VMD al ingreso en un Hospital de Asistencia a Crónicos de Media y Larga Estancia (HACMLE).

\section{MATERIAL Y MÉTODOS}

Muestra de pacientes: Estudiamos a 117 pacientes de edad igual o superior a 75 años ingresados en la Unidad de Convalecencia-Larga Estancia de un HACMLE de la Comunidad Valenciana.

Todos los pacientes fueron evaluados por medio de la historia clínica convencional propia de la Medicina Interna más una VMD (índice de Barthel, test de Pfeiffer, presencia problemática sociofamiliar, etc.) $(6,7)$ y mediante la determinación de algunos parámetros analíticos básicos.

Tipo de estudio: Longitudinal y prospectivo.

Parámetros a estudio: A todos los pacientes, dentro de las 48 horas posteriores a su ingreso, se les realizó una valoración multidimensional, destacando fundamentalmente: edad, sexo, diagnóstico principal y comorbilidad, factores de riesgo (sonda nasogástrica y/o vesical y perfusiones intravenosas), presencia de problema social, valoración del estado mental (test de Pfeiffer), y funcional (índice de Barthel), tanto al ingreso como al alta, número de fármacos al ingreso y al alta, presencia de complicaciones hospitalarias, actitud terapéutica tanto al ingreso como al alta. También se determinó al ingreso los valores correspondientes a variables bioquímicas como albúmina, colesterol total, linfocitos totales y hemoglobina.

Análisis de los datos:

—Del modelo de valoración multidimensional obtenemos las características de los pacientes y realizamos un análisis estadístico descriptivo de las variables más importantes.

- Realizamos un modelo de regresión logística (MRL) para ver la relación entre la variable dependiente fallecimiento durante la estancia hospitalaria, (categorizada de forma binaria: $0=$ no fallecimiento y $1=$ fallecimiento) y las variables explicativas $\mathrm{o}$ independientes: edad, enfermedad de base, comorbilidad, $\mathrm{n}^{\circ}$ de factores de riesgo, índice de Barthel y Pfeiffer al ingreso, variables analíticas antes reseñadas, etc. Pretendemos encontrar una regresión logística como modelo de probabilidad de que ocurra el suceso dado como valor 1 en la variable dependiente, teniendo en cuenta las variables explicativas antes indicadas.

-El análisis estadístico se ha realizado por medio del programa Statistical Package for the Social Sciencies PSS/PC+ 10.0

\section{RESULTADOS}

1. Variables descriptivos más importantes al ingreso (Tabla I).
TABLA I

VARIABLES DESCRIPTIVAS MÁS IMPORTANTES AL INGRESO $(\mathrm{N}=117$ PACIENTES)

\begin{tabular}{lr}
\hline Edad & $80 \pm 4$ \\
Sexo & \\
Hombres & $39 \%$ \\
Mujeres & $61 \%$ \\
Diagnóstico principal & \\
Infecciones & $10 \%$ \\
Demencias & $21 \%$ \\
AVC & $22 \%$ \\
ICC & $12 \%$ \\
EPOC & $21 \%$ \\
UPP & $7 \%$ \\
Otros & $7 \%$ \\
Comorbilidad & $4,2 \pm 1$ \\
Factores de riesgo & \\
0 & $20 \%$ \\
1 & $38 \%$ \\
$\geq 2$ & $42 \%$
\end{tabular}

I. de Barthel ingreso

$30,3 \pm 28,7$

$($ mediana $=25)$

Nivel cognitivo ingreso:

Normal

Deterioro leve

Deterioro moderado

Deterioro grave

Deterioro leve-moderado

Deterioro moderado-grave

No valorable

Albúmina ingreso

Colesterol total ingreso

Linfocitos ingreso

Hemoglobina ingreso

Problema social

Sí

No

$31 \%$

$13 \%$

$12 \%$

$31 \%$

$1,5 \%$

$2,5 \%$

$9 \%$

$3 \pm 0,6$

$172 \pm 48$

$1829 \pm 1011$

$12 \pm 2$

$19 \%$

$81 \%$

$N^{\circ}$ fármacos ingreso

$6,8 \pm 7,3$

Complicaciones hospitalarias

No

Neumonía

$47 \%$

21

ITU

11

Sepsis

Ins, Renal

Sindr, confusional

Otras

Estancia hospitalaria

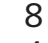

4

3

17

$35 \pm 32,5$

$($ mediana $=26)$

Motivo alta hospitalaria

Domicilio

$38,5 \%$

Residencia asistida

$15,4 \%$

Trasl. hospital agudos

Exitus

$6,8 \%$

$38,5 \%$

Voluntaria

0

Otros 
AVC: accidente vascular cerebral. ICC: insuficiencia cardiaca congestiva. EPOC: enfermedad pulmonar obstructiva crónica. UPP: úlceras por presión.

Llama la atención el predominio de mujeres (61\%), sobre hombres (39\%), la elevada prevalencia de deterioro neurológico (demencias graves y accidentes vasculares cerebrales como primer diagnóstico), lo que se explica por la avanzada edad de nuestros pacientes, y especialmente el alto porcentaje que ingresaba con dos o más factores de riesgo (42\%), entendiendo como tales, sondas, perfusiones y catéteres, fundamentalmente. Esto último probablemente obedezca al hecho de que casi todos nuestros enfermos venían remitidos desde Hospitales de agudos.

2. Modelo de regresión logística (MRL) (Tabla II).

En esta tabla se aprecia como el deterioro cognitivo (cuantificado por el test de Pfeiffer), la situación funcional (determinada por el índice de Barthel), y los niveles bajos de albúmina, se asocian de un modo estadísticamente significativo con una mayor mortalidad.

\section{DISCUSIÓN}

Los PEAP presentan unas determinadas características como comorbilidad, pérdida de la autonomía, discapacidad, alteraciones sensoriales, deterioro cognitivo y problemática sociofamiliar, que pueden presentarse simultáneamente, condicionando una especial dificultad.

Los programas de VMD pretenden detectar variables con el fin de mejorar la capacidad funcional y la supervivencia. La identificación de variables con valor pronóstico es útil para favorecer un uso racional de recursos y una adecuada toma de decisiones.

El pronóstico en Geriatría se establece fundamentalmente en base a tres grandes variables:

- Mortalidad.

- Capacidad funcional.

- Lugar de residencia (domicilio o institución).

La situación funcional es una variable muy importante en la valoración global de los pacientes ancianos y tiene poder predictivo de desarrollar dependencia, institucionalización, consumo de recursos sanitarios y sociales y mayor riesgo de mortalidad (8). La discapacidad se asocia con un incremento de la mortalidad y conlleva un aumento de resultados desfavorables como hospitalización, ingreso en residencia asistida y uso formal e informal de recursos sociales y sanitarios domiciliarios. Edad: a mayor edad, mayor fragilidad y comorbilidad y por tanto peor pronóstico.

Tiempo transcurrido desde el proceso causante de la disca- pacidad: a mayor tiempo transcurrido desde el inicio de la discapacidad, menor probabilidad de mejoría de la misma.

Diagnóstico principal: algunas enfermedades determinan intrínsecamente el carácter irreversible del deterioro del paciente (grandes síndromes neurológicos, amputaciones, etc.), pero en numerosas ocasiones, el diagnóstico principal aislado (como variable pronóstica) tiene un bajo poder predictivo, a no ser que se acompañe de otras variables que cuantifiquen la severidad del mismo.

Estado general de salud: incluye la presencia y/o coexistencia de enfermedades crónicas y tienen valor pronóstico sobre la funcionalidad, mortalidad y supervivencia (9).

Déficits sensoriales: por sí mismos o relacionados con la enfermedad o enfermedades de base, representan factores de mal pronóstico.

Estado nutricional: constituye un factor pronóstico capaz de influir en la recuperación de la capacidad funcional y en la mortalidad de los PEAP. La hipoalbuminemia es un factor predictivo de mortalidad; la disminución del nivel de colesterol y la disminución del perímetro muscular del brazo, son factores predictivos de la aparición de complicaciones en el PEAP (el deterioro del estado nutricional conlleva a un retraso en la curación úlceras, aumento de las infecciones, mayor mortalidad, etc.). En algún estudio se ha observado que existe una relación entre hipoalbuminemia y mayor riesgo de mortalidad en pacientes de edad avanzada. Este riesgo es aún mayor si junto a aquella se asocia un determinado nivel de discapacidad, por lo que no pueden servir de indicadores de fragilidad.

Deterioro cognitivo: constituye una variable de mal pronóstico para la rehabilitación por falta de colaboración y comprensión, y mayor riesgo de institucionalización (10).

Depresión: falta de motivación para la rehabilitación y cumplimentación de tratamientos.

Soporte socio-familiar: si es bueno influye produciendo una mejoría del pronóstico funcional. La falta de apoyo sociofamiliar constituye un factor predictivo de mortalidad.

La evaluación de todas estas variables es fundamental, tanto para emitir un pronóstico que permita tomar decisiones clínicas en los PEAP, como, para cuando sea posible, mejorar su pronóstico.

Los PEAP suelen ingresar en los hospitales a causa de enfermedades agudas que están asociadas con declive funcional, lo que puede conducir a la necesidad de cuidados de larga duración o a la muerte; en este último caso contribuyen también factores propios de la hospitalización (nosocomiales): comorbilidad, a veces no diagnosticada, yatrogenia y deterioro funcional.

Existen estudios randomizados que demuestran que una intervención adecuada de estos pacientes (prevención de com-

TABLA II

MODELO DE REGRESIÓN LOGÍSTICA (MRL)

\begin{tabular}{lccccccc}
\hline Variable & $B$ & E.T. & Wald & $d f$ & Sig & OR & IC (95\%) \\
\hline Comorbilidad & $-0,140$ & 0,247 & 0,320 & 1 & 0,571 & 0,870 & $0,748-0,992$ \\
Fact, de riesgo & $-0,169$ & 0,337 & 0,251 & 1 & 0,616 & 0,845 & $0,695-0,995$ \\
I, de Barthel & 2,471 & 0,943 & 6,867 & 1 & 0,009 & 11,833 & $9,32-13,893$ \\
T, de Pfeiffer & $-2,176$ & 1,105 & 3,877 & 1 & 0,049 & 0,113 & $0,05-0,153$ \\
Albúmina & $-1,758$ & 0,446 & 15,558 & 1 & 0,000 & 0,155 & $0,092-0,322$ \\
\hline
\end{tabular}


plicaciones y yatrogenia, movilización temprana, rehabilitación y programación del alta) disminuye la mortalidad (11); ésta también disminuye cuando se llevan a cabo programas de atención domiciliaria a los PEAP, si se basan en una valoración multidimensional y un seguimiento ambulatorio y siempre y cuando los pacientes ancianos tengan una relativa buena situación funcional basal, hipotésis basada en estudios de metanálisis (12). Las personas mayores con comorbilidad y niveles bajos de colesterol presentan menor probabilidad de supervivencia que las que presentan menos enfermedades concomitantes e hipocolesterolemia.

Determinados estudios demuestran que la depresión y actitudes negativas frente a la enfermedad conlleva a una disminución de la supervivencia tanto en pacientes con enfermedades malignas como enfermedades crónicas no malignas (13).

Necesidad de desarrollar escalas de predicción de la mortalidad: un estudio observacional realizado en un hospital de crónicos de EE.UU. y basado en la aplicación de un instrumento de valoración global de los pacientes crónicos por medio de un equipo multidisciplinar, que valora el "score" observado en los cambios de salud, enfermedad avanzada y síntomas y signos de problemas médicos (Changes in Health, End-stage disease and Symptoms and Signs: CHESS), demuestra como dicho "score" es un fuerte predictor de mortalidad y otras alteraciones asociadas con la fragilidad del PEAP (14).

En relación con los predictores bioquímicos de mortalidad, recientes estudios se han centrado sobre marcadores de la inflamación, que reflejan procesos patológicos de base, que pueden contribuir a aumentar el riesgo de mortalidad en las personas mayores. En los PEAP, inflamaciones episódicas o procesos inflamatorios crónicos juegan un papel no sólo en el daño vascular sino también en varios mecanismos de enfermedades, incluyendo la fragilidad y las enfermedades cardiovasculares (15).

En un estudio prospectivo de cohorte realizado en una unidad de geriatría muestra como el aumento en plasma, al ingreso hospitalario, de alfa-1-glicoproteína y de proteína Creactiva se asocian con un mayor riesgo de mortalidad durante la hospitalización, pero al realizar un análisis multivariante, sólo la alfa-1-glicoproteína permanece como predictor independiente de la mortalidad global (16); ésto está en relación con las bases biológicas del síndrome de fragilidad en los pacientes geriátricos que ha sido difícil de establecer dadas la falta de una definición exacta del mismo, su complejidad y la frecuente coexistencia con enfermedades, pero parece que los fundamentos fisiológicos de dicho síndrome se basan en la sarcopenia, procesos de disregulación neuroendocrina y en procesos inflamatorios que conducen a un declive funcional del paciente. Parece ser que tanto la inflamación, medida por el incremento de la proteína $\mathrm{C}$ reactiva, como marcadores de la coagulación medidos por un aumento del factor VIII y del dímero D, aumentan en pacientes ancianos con síndrome de fragilidad incluso cuando no se presentan diabetes ni enfermedad cardiovascular.

La supervivencia disminuye en los PEAP cuando sufren encamamiento tanto en hombres como en mujeres, pero sobre todo en los grupos de mayor edad (> 80 años).

En líneas generales, los factores pronósticos que se asocian a una mayor mortalidad, obtenidos mediante una minuciosa VMD durante la estancia hospitalaria de los PEAP en nuestro Hospital, son similares a los descritos en la literatura consultada.

\section{Bibliografía}

1. Zambrana García JL, Velasco Malagón MJ, Diez García F, Cruz Caparrós G, Martín Escalante MD, Adarraga Cansino. Characteristics of patients with multiple disease hospitalized in Internal Medicine Services. Rev Clin Esp 2005; 205: 413-7.

2. Petersen MA, Groenvold M, Aaronson N, Blazeby J, et al. for the European Organisation for Research and Treatment of Cancer Quality of Life Group. J Clin Epidemiol 2006; 59: 36-44.

3. Farquhar M, Grande G, Todd C, Barclay S. Defining patients as palliative: hospital doctor's versus general practitioners'perceptions. Palliative Medicine 2002; 16: 247-50.

4. Ponzetto M, Maero B, Maina P, Rosato R, Ciccone G, Merleti F, et al. Risk factors for early and late mortality in hospitalized older patients: the continuing importance of functional status. J Gerontol A Biol Sci Med Sci 2003; 58: 1049-54.

5. Navarro R, Juez P, Botella J, Espinar MV, Peris M, Errando E, Castillo M. Estudio de los factores pronósticos en pacientes geriátricos de un Hospital de Asistencia a Crónicos y Larga Estancia. Rev Esp Geriatría y Gerontología 1998; 33 (Supl. 1): 29.

6. Van Bennekom CAM, Jelles F, Lankhorst GJ, Bouter LM. Responsiveness of the Rehabilitation Activities Profile and the Barthel Index. J Clin Epidemiol 1996; 49: 39-44

7. Pfeiffer E. A short portable mental status questionnaire for the assessment of organic brain déficit in elderly patients. J Am Geriatr Soc 1975; 10: 433-41.

8. Miller DK, Andresen EM, Malmstrom TK, Miller JP, Wolinsky FD. Test-retest reliability of subclinical status for functional limitation and disability. J Gerontol B Psychol Sci Soc Sci 2006; 61: 52-6.
9. Corsonello A, Pedone C, Carosella L, Corica F, Mazzei B, Incalzi RA. Health status in older hospitalized patients with cancer or non-neoplastic chronic diseases. BMC Geriatr 2005; 25: 5-10.

10. McGuire LC, Ford ES, Ajani UA. Cognitive functioning as a predictor of functional disability in later life. Am J Geriatr Psychiatry 2006; 14 : 36-42.

11. Saltvedt I, Spigset O, Ruths S, Fayers P, Kaasa S, Sletvold O. Patterns of drug prescription in a geriatric evaluation and management unit as compared with the general medical wards: a randomised study. Eur J Clin Pharmacol 2005; 61: 921-8.

12. Stuck AE, Egger M, Hammer A, Minder CE, Beck JC. Home visits to prevent nursing home admission and functional decline in elderly people. Systematic review and meta-regression analysis. JAMA 2002; 287 : 1022-28.

13. Lewis SC, Dennis MS, O'Rourke SJ, Sharpe M. Negative attitudes among short-term stroke survivors predict worse long-term survival. Stroke 2001; 32: 1640-45.

14. Hirdes JP, Frijters DH, Teare GF. The MDS-CHESS Scale: A new measure to predict mortality in institutionalized older people. J Am Geriatr Soc 2003; 51: 96-100.

15. Newmann AB, Gottdiener JS, McBurnie MA, et al. For the Cardiovascular Heath Study Research Group. Associations of subclinical cardiovascular disease with fraility. J. Gerontol Med Sci 2001; 56 A: M15866.

16. Henry OF, Blacher J, Verdavaine J, Duviquet M, Safar ME. Alpha 1 acid glycoprotein is an independent predictor of in hospital death in the elderly. Age Ageing 2003; 32: 37-42. 